\title{
A cosmological view on Milgrom's acceleration constant
}

\author{
Engel Roza \\ Philips Research Labs, Eindhoven, The Netherlands (retired) \\ Email: engel.roza@onsbrabantnet.nl
}

\begin{abstract}
Summary
In this article it is shown how Milgrom's acceleration constant is related with the $\Omega_{\Lambda}, \Omega_{m}$ and $\Omega_{B}$ values of the matter distribution within observable universe as defined in the Standard Model of Cosmology, established by the Planck Collaboration Group. The result is a two-parameter model for the observable universe. The two parameters are the age of the universe and Milgrom's acceleration constant. It is shown that these are sufficient to calculate the amounts of matter and dark energy in the universe, as well as the contributions of dark matter and baryonic matter in the matter part.. The numerical results are in agreement with those of the Lamda-CDM model.
\end{abstract}

Keywords: Cosmological Constant; MOND; dark matter; dark energy; Lamda-CDM model

\section{Introduction}

It is well known that, as long as the Cosmological Constant $\Lambda$ is supposed to be zero, the Newtonian potential field $\Phi$ can be derived as the weak field limit of Einstein's Field Equation. Although a nonzero value of $\Lambda$ is a major roadblock to derive an expression for a modified Newtonian potential, it can be done under particular constraints for the spatial validity range. A previous study [1] shows that, under these conditions, the resulting potential $\Phi$ of cosmological systems with a central pointlike mass $M$ is the solution of the field equation,

$\frac{\partial^{2}}{\partial r^{2}}(r \Phi)+\lambda^{2}(r \Phi)=-r \frac{4 \pi G M}{c^{2}} \delta^{3}(r)$, where $\lambda^{2}=2 \Lambda$,

which in an alternative format can be written as,

$$
\nabla^{2} \Phi+\lambda^{2} \Phi=-\frac{4 \pi G M}{c^{2}} \delta^{3}(r)
$$

where $\nabla^{2}$ is the Laplacian operator and $G$ and $c$, as usual are, respectively, the gravitational constant and the light velocity in vacuum. The constraints mentioned apply to the extreme low end of the spatial range as well as to the extreme far end of it. The first constraint is not different from the weak field limitation that has to be imposed to derive Poisson's equation in the case of $\Lambda=0$. The second constraint is required to allow the derivation of $\Phi$ from a single metric component in Einstein's metric tensor. As shown in [1], these conditions are met in solar systems as well as in galaxy systems.

The striking feature of $(1,2)$ is the + sign associated with $\lambda^{2}$. If it were a - sign, the equation would be similar to Debije's equation for the potential of an electric pointlike charge in an electromagnetic 
plasma [2]. As is well known, the solution of such equation is a shielded Coulomb field, i.e., an electric field with an exponential decay. In the gravitational equivalent (with the + sign) the near field is enhanced ("antiscreened"), because masses are attracting, while electric charges with the same polarity are repelling. The way to solve the equation, though, is similar. The solution of this equation shows that, in the relevant spatial range, the gravitational acceleration $g$ closely approximates Milgrom's heuristic expression [3],

$g=\sqrt{g_{N} a_{0}}$,

where $g_{N}$ is the common Newtonian gravitation and where $a_{0}$ is Milgrom's acceleration constant. The study [1] has shown that, for spherical cosmological systems with a central pointlike central mass $M$, this acceleration is related with the Cosmological Constant in those systems, such that

$\lambda^{2}=2 \Lambda=\frac{2 a_{0}}{5 M G}$

It is my aim to show that this relationship between Milgrom's acceleration constant and the Cosmological constant not only holds for galaxies, but also holds for the observable universe within the event horizon, in spite of the obvious difficulty to identify a central mass. The study is prompted by the wish to find a means to assess the numerical value of Milgrom's acceleration constant by theory, for which no clue could be found within the scope of galaxies. Doing so, it is useful to emphasize that, unlike Newton's gravitational constant $G$, Einstein's Cosmological Constant $\Lambda$ is not an invariable, but depends on the amount of baryonic mass $M$ in the system under consideration. Baryonic mass is just a single component of gravitational matter. The gravitational matter density $\rho c^{2}$ shows up in the vacuum solution of Einstein's Field Equation as the background energy of a vacuum fluid in thermal dynamical equilibrium. The vacuum fluid is an ideal one. This implies that its stress-energy tensor contains diagonal elements only and that in space-time (ict, $x, y, z)$ with $(+,+,+,+)$ metric they all have the same value. The pressure $p_{v}$ is due to the background energy of the fluidal space, expressed by $[4,5,6,7]$,

$p_{v}=-\rho c^{2}=-\frac{c^{4}}{8 \pi G} \Lambda$

where $\rho$ is the fluidal mass density. It must be present for giving a solution of Einstein's Field Equation with $\Lambda \neq 0$ for the vacuum without any baryonic sources. Assuming the correctness of (4), the fluidal pressure would rise to infinity under the absence of baryonic source. Baryonic sources "eat" from the fluid, thereby gaining mass and reducing the energy level of the fluid. This model is applicable to cosmological objects with a massive kernel, like galaxies. Interestingly, though, it can be evolved to a model that fits to the cosmos where matter is distributed. The gravitational matter density is composed by various components. In accordance with the nomenclature in the LamdaCDM model $(C D M=$ Cold Dark Matter, Lamda $=\Lambda)$ [8], we have,

gravitational matter $=$ (true) matter + dark energy matter

(true) matter = baryonic matter + dark matter.

Normalizing all densities on the gravitational matter density gives, 
$1=\Omega_{m}+\Omega_{\Lambda}=\left(\Omega_{B}+\Omega_{D}\right)+\Omega_{\Lambda}$

where $\Omega_{m}, \Omega_{\Lambda}, \Omega_{B}, \Omega_{D}$, respectively are the relative matter density, the relative dark energy matter density, the relative baryonic matter density and the relative dark matter density. These symbols are the ones used in the Lamda-CDM. It will be shown in this article, that the interpretation of the Cosmology Constant concept as expressed by (4), developed from a straight evolution from Einstein's Field Equation [9], nicely fits to the Lamda-CDM model that evolves from the FriedmannLemairtre-Robertson-Walker (FLRW) metric $[10,11]$. One of the results is the assessment of a numerical value of Milgrom's acceleration constant by theory. Other benefits are a better understanding of the nature of dark matter and dark energy and a simple rudimentary twoparameter model for cosmology.

\section{Cosmological model}

Let us model the visible universe as a sphere within the event horizon $L$. It contains distributed gravitational energy. This distributed energy is a gradually developed mixture of the energy from fluidal matter as meant by (5) and the energy from baryonic matter $M_{B}$ as meant by (4). Hence, from (4) and (5),

$$
M_{G} c^{2}=\int_{0}^{L} \frac{a_{0}}{5 M_{B} G} \frac{c^{4}}{8 \pi G} 4 \pi r^{2} d r
$$

Let $\Delta M_{G}$ the difference between the gravitational matter in a sphere $L+\Delta L$ and the gravitational matter in a sphere $L$. It follows readily that

$$
c^{2} \Delta M_{G}=\frac{a_{0}}{10 M_{B} G^{2}} c^{4} L^{2} \Delta L
$$

After writing the baryonic matter as a dimensionless fraction $\Omega_{B}$ of the gravitational matter as,

$$
M_{B}=\Omega_{B} M_{G},
$$

eq. (8) can be integrated as

$$
\frac{1}{2} M_{G}^{2} c^{2}=\frac{a_{0}}{10 \Omega_{B} G^{2}} c^{4} \frac{L^{3}}{3} .
$$

The gravitational energy $M_{G} c^{2}$ therefore, is

$$
M_{G} c^{2}=\sqrt{\frac{a_{0}}{5 \Omega_{B} G^{2}} c^{6} \frac{L^{3}}{3}} .
$$

Hence, the gravitational mass density $\rho_{G}$ in the sphere with radius $L$ is given by

$$
\rho_{G}=\frac{1}{c^{2}} \sqrt{\frac{a_{0}}{5 \Omega_{B} G^{2}} c^{6} \frac{L^{3}}{3}} \cdot \frac{3}{4 \pi L^{3}}=\frac{3}{4 \pi c^{2}} \sqrt{\frac{a_{0}}{15 \Omega_{B} G^{2}} \frac{c^{6}}{L^{3}}} .
$$


This relationship between baryonic energy and gravitational energy allows us to compare the gravitational energy with the one from the Lamda-CDM model. In that model, the gravitational mass density is known as the critical mass density $\rho_{c}$. The CDM-Lamda model has been evolved from the Einstein-de Sitter model that has been the preferred one for the universe up to the $1980 \mathrm{~s}$. It has been refined to the present one to cope with certain cosmological phenomena, like for instance the discovery of the accelerating universe in 1998, $[12,13,14]$. For further evaluation of (12) to arrive at a numerical value of Milgrom's acceleration $a_{0}$, it will be useful to consider a recapitulation the Lamda-CDM model as presented in the next section.

\section{The Lamda-CDM model}

The model evolves from the solution of Einstein's Field Equation under the constraint of a particular metric.

$G_{\mu v}+\Lambda g_{\mu v}=\frac{8 \pi G}{c^{4}} T_{\mu v} \quad$ with $G_{\mu v}=R_{\mu \nu}-\frac{1}{2} R g_{\mu \nu}$

where $T_{\mu \nu}$ is the stress-energy tensor, which describes the energy and the momenta of the source(s) and where $R_{\mu v}$ and $R$ are respectively the so-called Ricci tensor and the Ricci scalar, which can be calculated if the metric tensor components $g_{\mu \nu}$ are known $[15,16]$. The adopted metric, known as the FLRW metric [11], is

$$
\mathrm{d} s^{2}=\mathrm{d} q_{0}^{2}+a^{2}(t)\left(\frac{\mathrm{d} r^{2}}{1-k r^{2}}+r^{2} \sin ^{2} \vartheta \mathrm{d} \varphi^{2}+r^{2} \mathrm{~d} \vartheta^{2}\right)
$$

where $q_{0}=\mathrm{ict}$ is the normalized time coordinate $(\mathrm{i}=\sqrt{-1})$, and where $k$ is a measure for the curving of space-time. The scale factor $a(t)$ expresses the time-dependence of the size of the universe. The ratio

$$
\frac{\dot{a}}{a}=H(t)
$$

is known as the Hubble factor. It is the main observable of the universe, because its numerical value can be established from red shift observations on cosmological objects ( $\dot{a} \equiv \mathrm{d} a / \mathrm{d} t)$.

By moving the term $\Lambda g_{\mu \nu}$ to the right side of (13), it can be conceived as an additional contribution to the energy density,

$$
\begin{aligned}
& \rho(t) \rightarrow \rho(t)+\rho_{\Lambda} ; \quad \rho_{\Lambda}=\frac{\Lambda}{\kappa} ; \kappa=\frac{8 \pi G}{c^{2}} . \\
& p(t) \rightarrow p(t)-\rho_{\Lambda} c^{2} .
\end{aligned}
$$


Note: the difference in sign between the $\rho_{\Lambda}$ terms in (16a) and (16b) is due to the choice of the metric as ( (ict, $x, y, z)$ with $(+,+,+,+)$.

The solution of (13) under constraint of the metric (14) are the two Friedmann equations [10],

$$
\begin{aligned}
& \left(\frac{\dot{a}}{a}\right)^{2}+\frac{k c^{2}}{a^{2}}=\frac{8 \pi G}{3} \rho \\
& 2 \frac{\ddot{a}}{a}+\left(\frac{\dot{a}}{a}\right)^{2}+\frac{k c^{2}}{a^{2}}=-\frac{8 \pi G}{c^{2}} p .
\end{aligned}
$$

Under the constraint $k=0$ (flat universe), and taking into consideration (16a,b), the first Friedmann equation evolves as,

$$
\left(\frac{\dot{a}}{a}\right)^{2}=\frac{8 \pi G}{3}\left(\rho+\rho_{\Lambda}\right) \rightarrow\left(\frac{\dot{a}}{a}\right)^{2}=\frac{8 \pi G}{3} \rho_{t} ; \quad \rho_{t}=\rho+\rho_{\Lambda}
$$

The second Friedmann equation reads as,

$$
\frac{\ddot{a}}{a}=\frac{4}{3} \pi G\left(2 \rho_{\Lambda}-\rho\right) \rightarrow \frac{\ddot{a}}{a}=\frac{4}{3} \pi G\left(2 \rho_{\Lambda}+2 \rho-3 \rho\right)=\frac{8}{3} \pi G \rho_{t}\left(1-\frac{3}{2} \alpha\right) ; \alpha=\frac{\rho}{\rho_{t}} .
$$

Differentiating the mass density $\rho_{t}$ in (18a) gives,

$$
\begin{aligned}
& \dot{\rho}_{t}=\frac{3}{8 \pi G} \frac{\mathrm{d}}{\mathrm{d} t}\left(\frac{\dot{a}}{a}\right)^{2}=\frac{3}{8 \pi G}\left[2 \frac{\dot{a}}{a}\left\{\frac{\ddot{a}}{a}-\left(\frac{\dot{a}}{a}\right)^{2}\right\}\right]= \\
& 2 \frac{3}{8 \pi G} \frac{\dot{a}}{a}\left\{\frac{8}{3} \pi G\left(1-\frac{3}{2} \alpha\right)-\frac{8 \pi G}{3}\right\} \rho_{t}=2 \frac{\dot{a}}{a}\left\{\left(1-\frac{3}{2} \alpha\right)-1\right\} \rho_{t}=-3 \alpha \frac{\dot{a}}{a} \rho_{t} \rightarrow \\
& \dot{\rho}_{t}=-3 \alpha \frac{\dot{a}}{a} \rho_{t} \rightarrow \frac{1}{\rho_{t}} \frac{\mathrm{d} \rho_{t}}{\mathrm{~d} t}=-3 \frac{\rho}{\rho_{t}} \frac{1}{a} \frac{\mathrm{d} a}{\mathrm{~d} t} \rightarrow \frac{\mathrm{d} \rho_{t}}{\mathrm{~d} t}=-3 \rho \frac{1}{a} \frac{\mathrm{d} a}{\mathrm{~d} t} \rightarrow \\
& \frac{\mathrm{d} \rho}{\mathrm{d} t}+\frac{\mathrm{d} \rho_{\Lambda}}{\mathrm{d} t}=-3 \rho \frac{1}{a} \frac{\mathrm{d} a}{\mathrm{~d} t} .
\end{aligned}
$$

Because $\rho_{\Lambda}$ is time-independent ( $\Lambda$ is independent of space-time coordinates), (19) is satisfied if,

$$
\frac{\mathrm{d} \rho}{\mathrm{d} t}=-3 \rho \frac{1}{a} \frac{\mathrm{d} a}{\mathrm{~d} t} \rightarrow \rho=\Omega_{m} H_{0} a^{-3} \text { and } \rho_{\Lambda}=H_{0} \Omega_{\Lambda} \text {, }
$$

where $\Omega_{m}, \Omega_{\Lambda}$ and $H_{0}$ are constants. Although satisfying the equation would need two arbitrary constants only, a third one is added to impose the additional constraint

$$
\Omega_{m}+\Omega_{\Lambda}=1
$$

Applying $(20 a, b)$ on the first Friedmann equation (18a), results into, 


$$
H(a)=\frac{\dot{a}}{a}=H_{0} \sqrt{\Omega_{m} a^{-3}+\Omega_{\Lambda}} .
$$

The quantity $H_{0}$ can now be interpreted as the Hubble parameter $\dot{a} / a$ at $a(t)=1$.

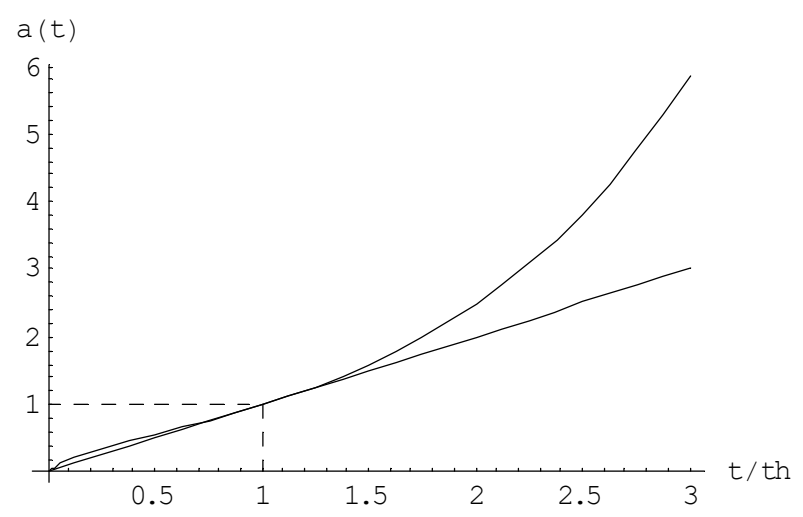

Fig.1: The scaling factor $a(t)$ as a function of cosmological time. The lower curve represents Hubble's law. The upper curve shows the curve of accelerated scaling due to Einstein's Cosmological Constant.

Eq. (22) represents the Lamda-CDM model in its most simple format (actually, more terms are heuristically added under the square root operator to model empirical evidence from certain cosmological phenomena). It can analytically be solved as [17],

$$
a(t)=\left(\frac{\Omega_{m}}{\Omega_{\Lambda}}\right)^{1 / 3} \sinh ^{2 / 3}\left(3 \sqrt{\Omega_{\Lambda}} t / 2 t_{H}\right) ; t_{H}=H_{0}^{-1} .
$$

At present time $t=t_{P}$, the scale factor equals unity $(a=1)$ and the Hubble parameter is the observable $H_{0}$. Equating present time $t_{P}$ with Hubble time $t_{H}=H_{0}^{-1}$ is justified if $a(t)$ would have shown a linear increase over time up to now, under a constant rate of say $c_{0}$, because in that case $a(t)=c_{0} t$ and $\dot{a}(t)=c_{0}$. This is Hubble's empirical law. Equating $t_{P}=t_{H}$ in (23) as an axiomatic assumption, indeed results in a behavior of the scale curve that, up to present time $t \leq t_{H}$, is pretty close to Hubble's empirical law. As imposed, from (23),

$a\left(t_{H}\right)=1 ; H(a) t_{H}=H_{0} \rightarrow \Omega_{m}+\Omega_{\Lambda}=1$

Hence, from (23) and (24),

$\left(\frac{1-\Omega_{\Lambda}}{\Omega_{\Lambda}}\right)^{1 / 3} \sinh ^{2 / 3}\left(3 \sqrt{\Omega_{\Lambda}} / 2\right)=1 \rightarrow \Omega_{\Lambda}=0.737 ; \Omega_{m}=0.263$

These values are only slightly different from those in the six-parameter Lamda-CDM model (where $\Omega_{m}=0.259$ ). The difference is due to the simplicity of the format (22). Figure 1 demonstrates the viability of the axiomatic assumption to equate present time with Hubble time. Apparently, from comparing (24) with $(18 \mathrm{a})$ and $(20 \mathrm{a}, \mathrm{b})$, it appears that the total gravitational matter is built up by a 
matter part $\Omega_{\Lambda}$ as a consequence of the presence of the Cosmological constant and a matter part $\Omega_{m}$ from the residual fluidal background energy.

It may seem that, in retrospect, the scaling in the past has been dominated by $\Omega_{m}$ and that it showed an almost linear increase of the scaling factor $a(t)$ with cosmological time, while the future scaling may seem being dominated by $\Omega_{\Lambda}$, thereby no longer showing a linear increase. However, an observer in the far past would have drawn the very same conclusion and an observer in the far future will do as well, because there is no reason why he/she should not adopt the same view on Einstein's Equation, the metric and Hubble's empirical law. Moreover, curiously, the simple fact of adopting a Cosmological Constant of any arbitrary magnitude will lead to the same distribution between $\Omega_{\Lambda}$ (relative amount of dark energy) and $\Omega_{m}$ (relative amount of matter). This phenomenon can be explained by taking into consideration that the major impact on the model is due to the adoption of background energy from a fluidal space with a perfect symmetric stress-energy tensor and a tiny Cosmological Constant not affecting the symmetry of the FLRW-metric. These adoptions are the same for any cosmological observer, either in the present, either in the past, either in the far future.

The total gravitational mass $\rho_{t}(a=1)$, known as critical mass $\rho_{c}$ in the Lamda-CDM model observed within the Hubble horizon $L_{H}=c t_{H}$, can be established from (18a) as,

$\rho_{c}=\frac{3 H_{0}^{2}}{8 \pi G}$

The assumption that Hubble time is equal to present time allows to equate the Hubble horizon with the event horizon of the visible horizon. Hence,

$$
L=c t_{H}=c H_{0}^{-1}
$$

From (25) and (26), we have

$$
\rho_{c}=\frac{3}{8 \pi G L^{2}}
$$

At this point, we are able to compare the gravitational mass density (27) as developed in the LamdaCDM model with the gravitational mass density (12) as developed in the cosmological model of the second paragraph. They should be the same. The comparison will be made in the next paragraph.

\section{Comparing the Cosmological Model with the Lamda-CDM model}

Equating (12) with (27) gives,

$$
\frac{3}{8 \pi G L^{2}}=\frac{3}{4 \pi c^{2}} \sqrt{\frac{a_{0}}{15 \Omega_{B} G^{2}} \frac{c^{6}}{L^{3}}} .
$$

Elementary algebraic evaluation shows, 


$$
a_{0}=\frac{3}{4} \Omega_{B} a_{L} ; a_{L}=\frac{c^{2}}{L} .
$$

The quantity $a_{L}$ has the dimension of acceleration. From $L=c t_{H}$ and $t_{H}=13.8$ Gyear (Hubble time), we have $a_{L} \approx 6.9 \times 10^{-10} \mathrm{~ms}^{-2}$. The fraction $\Omega_{B}$ is known from the established empirical values in the Lamda-CDM model. In the preceding paragraph we have seen that the distribution between $\Omega_{m}$ and $\Omega_{\Lambda}$ is determined by theory. This is not the case as yet for the fraction $\Omega_{B}$. Although the theory developed in this article shows an intimate relationship between $a_{0}$ and $\Omega_{B}$, it is unable to reveal the quantitative distribution between those quantities. For determining the value of Milgrom's acceleration parameter $a_{0}$, the best that can be done is accepting the empirical value of $\Omega_{B}$ as established in the Standard Model of Cosmology from the Planck Collaboration Group, which says that [8],

$\Omega_{B}=\Omega_{m} \frac{\Omega_{B}}{\Omega_{m}}=0.259 \times 0.185=0.0486$

Inserting this value into (29) eventually gives,

$a_{0}=1.256 \times 10^{-10} \mathrm{~m} / \mathrm{s}^{2}$.

\section{Conclusion}

It has been shown how the numerical value of Milgrom's acceleration constant is related with the with the $\Omega_{\Lambda}, \Omega_{m}$ and $\Omega_{B}$ values of the matter distribution within observable universe as defined in the Standard Model of Cosmology as established by the Planck Collaboration Group. More in particular, it has been shown that

$a_{0}=\frac{3}{4} \Omega_{B} \frac{c}{t_{H}}$,

where $t_{H}$ is the Hubble timescale, which is commonly established as $t_{H} \approx 13.8$ Gyear. Furthermore, it has been shown that, by adopting the Hubble timescale and Milgrom's acceleration constant as primary parameters, the matter distribution of the observable universe over dark energy $\left(\Omega_{\Lambda}\right)$, matter $\left(\Omega_{m}\right)$, baryonic matter $\left(\Omega_{B}\right)$ and dark matter $\left(\Omega_{m}-\Omega_{B}\right)$ is fully determined as a theoretical consequence from combining the view from the FRLW-metric based Lamda-CDM model with the view on the anti-screening effect from the Cosmological Constant [1]. The result is a simple two-parameter model for the observable universe, which captures the major cosmological parameters.

\section{References}

[1] ] E. Roza, doi:10.20944/preprints201705.0164.v4 (2017)

[2] P. Debye and E. Huckel, Physik. Zetschrift, vol. 24, 9, 185 (1923) 
[3] M. Milgrom, The Astrophysical Journal, 270, 365 (1983)

[4] A. Einstein, Preuss. Akad. Wiss, Berlin (Math. Phys.), 142 (1917)

[5] www.scholarpedia.org/article/Cosmological constant

[6] S. Carroll, W. Press and E. Turner, Ann. Rev. Astronomy and Astrophys, 30, 499 (1992)

[7] J. Sola, Journal of Physics, Conf. Series, 453, 012015 (2013)

[8] Planck Collaboration, Astronomy and Astrophys., 594: A13 (2015)

[9] A. Einstein, Relativity: The Special and General Theory, H. Holt and Company, New York (1916, translation 1920)

[10] A. Friedmann, Zeitschrift fur Physik A, 10(1), 377 (1922)

[11] R. d'Inverno, Introducing Einstein's Relativity, Ch. 23, Oxford Press (1992)

[12] J.A. Frieman, M.S. Turner, D. Huterer, Dragan, Ann. Rev. Astronomy and Astrophys. 46, 385 (2008)

[13] G. Goldhaber, AIP Conference Proceedings 1166, 53, arXiv:0907.3526, (2009)

[14 P.J.E. Peebles, B. Ratra, Bharat (2003). Reviews of Modern Physics. 75 (2): 559, (2003)

[15] S. Weinberg, Gravitation and Cosmology, John Wiley \& Sons, Inc., New York (1972)

[16] T.A. Moore, A General Relativity Workbook , University Science Books, (2013)

[17] S. Wolfram, Mathematica, Addison Wesley, Redwood City A (1991) 